\title{
Etude écologique du Parc National de Mozogo-Gokoro (Cameroun): prospections préliminaires de la flore ligneuse et du sol pour sa conservation et son aménagement
}

\author{
Rodrigue Constant SANDJONG SANI ${ }^{1^{*}}$, Mama NTOUPKA ${ }^{2}$, Adamou IBRAHIMA ${ }^{3}$ et \\ VROUMSIA TOUA ${ }^{4}$
}

\author{
${ }^{1}$ Institut Supérieur du Sahel, Université de Maroua, BP 46 Maroua, Cameroun. \\ ${ }^{2} I R A D$ Forêt, BP 222 Maroua, Cameroun. \\ ${ }^{3}$ Université de Ngaoundéré, Faculté des Sciences, Département de Sciences Biologiques, \\ B.P. 454 Ngaoundéré, Cameroun. \\ ${ }^{4}$ Université de Maroua, Ecole Normale Supérieure, Département de SVT-BP 55 Maroua, Cameroun. \\ * Auteur correspondant, E-mail: sanirocos@yahoo.fr, Tel. +23775069143/+23794827869
}

\section{RESUME}

La conservation et la gestion rationnelle des aires protégées requièrent des études préalables de caractérisations écologique et floristique. Dans cette optique, il a été envisagé des prospections de base de la flore ligneuse et du sol pour l'aménagement du Parc National de Mozogo-Gokoro (PNMG), situé en zone sahélo-soudanienne, région de l'Extrême-Nord au Cameroun. Les données obtenues décrivent un sol favorable à la production végétale : texture sablo-limoneuse, activités biologiques présentes et absence d'érosion. La végétation présente une physionomie de forêt sèche claire à dense. La diversité compositionnelle montre sa grande richesse floristique, avec 62 espèces de ligneux identifiées. L'indice de Shannon est évalué à 3,75 bits avec une équitabilité de 0,84 . Dans sa structure, il peut être relevé une densité très élevée (2972 tiges/hectare) et la forte représentativité des moyennes et hautes tiges, preuves de sa grande préservation. Les types phytogéographiques rapprochent la végétation des zones soudaniennes à guinéennes. Ces caractéristiques peuvent lui conférer le statut d'écosystème de référence montrant un transfert d'espèces des zones guinéennes et soudaniennes au sahélien, malgré les menaces anthropiques notées. Ces spécificités floristiques peuvent s'expliquer par les mesures de conservation appliquées, la pédologie et la topographie (bas-fond, piémont). L'obtention d'attributs vitaux, de façon plus approfondie, pourrait confirmer ces analyses et contribuer plus significativement à son processus d'aménagement.

(c) 2013 International Formulae Group. All rights reserved.

Mots clés : Pré-inventaire, diversité compositionnelle, structure, écosystème de référence, menaces anthropiques, aire protégée.

\section{INTRODUCTION}

Ces dernières années avec l'influence de plusieurs traités internationaux sur les concepts d'environnement et de développement durable, la gestion rationnelle des ressources naturelles est devenue une priorité pour la plupart des pays de la planète. Le Cameroun n'est pas en marge de ce contexte de mobilisation internationale, avec l'adoption des textes législatifs tels que la loi 
forestière en 1994, la loi-cadre sur l'environnement en 1996 et la mise en œuvre d'une politique forestière conséquente (plusieurs programmes de gestion forestière orientés vers la durabilité).

Les parcs nationaux, constituants des aires protégées du domaine forestier permanent, doivent être gérés suivant un plan d'aménagement selon la nouvelle législation forestière. Le Nord-Cameroun, qualifié de zone à écologie fragile ou sensible, est soumis à une dégradation du milieu naturel imputable à la conjonction des facteurs climatiques, pédologiques et humains (Donfack et al., 1996 ; Djoufack-Manetsa, 2011). Pour la plupart des écosystèmes de la zone, la conservation nécessite des actions de réhabilitation, de restauration, voire de réaffectation. Concernant les perturbations anthropiques (coupe de bois, feux, pâturage), elles sont susceptibles de modifier le processus de l'évolution des formations végétales, tout en fragilisant leur relatif équilibre dynamique (Ntoupka, 1999 ; Hiernaux et Le Houérou, 2006). Plusieurs risques climatiques et édaphiques déterminent cette stabilité de la végétation, avec un impact sur le plan agronomique (Mbiandou'n et al., 2002; Hiernaux et Le Houérou, 2006). Les sols de la région sont ainsi dits fragiles (Brabant et Gavaud, 1985).

Qu'il s'agisse des zones de forêts ou de savanes, la gestion de la plupart des grands ensembles de conservation est pratiquée sur une base empirique sans réelle prise en compte des données scientifiques (Donfack, 2009). La délimitation des aires protégées dérive surtout de l'observation des traits physionomiques et faunistiques de la végétation sans caractérisations écologique et floristique. Avec la croissance démographique, des conflits sont perceptibles autour de ces zones protégées entre l'administration forestière et la population. Le présent travail a pour objectif global de contribuer à la conservation de la biodiversité végétale avec un intérêt pour les processus écologiques essentiels et le soutien d'une gestion rationnelle des ressources forestières. Pour un développement rural durable en Afrique subsaharienne, il est urgent de disposer d'une connaissance détaillée des ressources disponibles, notamment dans les forêts et terres boisées, de les planifier et les gérer (FAO, 2010). Il est envisagé dans cette étude, l'acquisition de données écologiques du Parc National de Mozogo-Gokoro (PNMG) en perspective d'une étude plus complète pour un plan d'aménagement durable. Il s'agit de décrire l'écosystème constitué par le parc sur la base de quelques paramètres écologiques, d'estimer la diversité compositionnelle et structurale sur le plan floristique, de déterminer le degré d'endémisme sahélosoudanien, d'identifier les signes de perturbations anthropiques et les indices d'appartenance à l'écosystème de référence favorables à sa conservation et son aménagement.

\section{MATERIEL ET METHODES \\ Zone d'étude}

La zone d'étude, le PNMG, est localisée dans la région administrative camerounaise de l'Extrême-Nord, département du Mayo-Tsanaga, arrondissement de Mayo-Moskota, entre 10 ${ }^{\circ} 56^{\prime}$ à $10^{\circ} 96^{\prime}$ de latitude Nord et $13^{\circ} 54^{\prime}$ à $13^{\circ} 58^{\prime}$ de longitude Est, d'une superficie de 1400 hectares (MINEF, 2000). Ce site a été créé comme réserve forestière et de faune par arrêté $\mathrm{N}^{\circ} 165$ du 12 Juin 1932 du Hautcommissaire de la République française au Cameroun et, érigé en parc national par arrêté $N^{\circ} 120$ du 05 décembre 1968 du Secrétariat au Développement de l'Etat du Cameroun (Wafo, 2008).

Le milieu physique du parc se situe au niveau des piémonts des Monts Mandara. Il appartient à l'unité géomorphologique englobant les plaines du Diamaré, de Mora et de Kaélé (Donfack et al., 1996). Le paysage est caractérisé par la présence de pédiments et glacis provenant des montagnes (Donfack et al., 1996). La carte établie par Brabant et Gavaud (1985) indique la présence, dans la 
zone, des sols évolués tels que les planosols et solonetz gleyiques sur granite d'anatexie et Gneiss, des sols peu évolués exondés des alluvions anciens des cours d'eau temporaires (Mayo), ainsi que des sols inondés de plaine de décantation argileuse (vertisols hydromorphes). La topographie est presque uniforme, avec une altitude variant peu autour de $450 \mathrm{~m}$ et une absence d'affleurement rocheux. Un cours d'eau non permanent, le Mayo-Nguetchewe traverse la végétation du parc du Nord au Sud. L'unité géomorphologique périphérique constituée par les monts Mandara et la végétation y instaure un climat plus doux, caractéristique de la zone soudano-sahélienne, avec une pluviosité comprise entre 800 et $1000 \mathrm{~mm}$ (L'Hote, 1998).

D'après les travaux de Letouzey (1985), la végétation peut présenter des imbrications de formations sahélosoudaniennes ou soudano-sahéliennes, savanicoles ou steppiques, avec plusieurs faciès de dégradation. La dynamique de cette végétation est liée à la présence d'une faune plus ou moins variée. Elle est constituée de mammifères et d'oiseaux (114 espèces) de groupes variés, de nombreux reptiles (Varans, Pythons canins...) et plusieurs batraciens et invertébrés (MINEF, 2000). Une population humaine importante (densité de 90,8 habitants au $\mathrm{km}^{2}$ dans la région de l'Extrême-Nord indiquée par le rapport du troisième recensement général de la population de 2005) et d'origines diverses (Mafa, Mandara, Kanuri, Glavda, Peuls) se retrouve dans la zone riveraine.

\section{Pré-inventaire et collecte des données}

L'exploitation des espèces ligneuses étant une menace directe pour la stabilité des écosystèmes sahélo-soudaniens, cette étude de base se fonde sur un pré-inventaire de ce type de ressource. Il s'est effectué à un très faible taux de sondage $0,06 \%$ car, d'après
Bellefontaine et al. (1997), dans les travaux d'aménagement forestier, un échantillonnage préliminaire à très faible taux de sondage est à préconiser, afin de caractériser l'hétérogénéité de la ressource et d'estimer le nombre minimum de placettes nécessaires pour atteindre une précision donnée. Trois placettes ont été ainsi localisées au cœur de la végétation, la placette $\mathrm{P} 1 \quad\left(10^{\circ} 56^{\prime} 54.57^{\prime}\right.$ ' $\mathrm{N}$, $13^{\circ} 55^{\prime} 24.95^{\prime}$ 'E) près de la localité de Karazawa et du Mayo Nguetchewé, la placette P2 (1057'59.16'’ N, 1356'00.70'’E) dans une zone considérée comme ayant abritée un ancien village, et la placette P3 (1057'42.72'’N, 1354'42.01'’E), localisée près de la localité de Mozogo. La Figure 1 donne une idée de l'emplacement des placettes.

Les descripteurs des espèces ligneuses sont obtenus dans ces placettes de $30 \mathrm{~m}$ de rayon. Une équipe de 6 personnes a été nécessaire : un pointeur qui remplit les fiches d'inventaire; trois observateurs mobiles considérés comme guides, aidant à l'obtention de mesures (hauteurs et circonférences) et à l'identification des espèces en langue vernaculaire; deux autres observateurs qui contrôlent les limites de la placette, aident au repérage, au décongestionnement, à l'étiquetage et à la récolte d'échantillons pour identification à l'Herbier National ou à partir des clés disponibles. Cette méthode de collecte de données floristiques est semblable à celle utilisée par Ntoupka (1999). Les données pédologiques simples sont relevées sur le terrain. Il s'agit de la texture du sol déterminée par des tests de manipulations manuelles successives de terrain (test de pression à partir d'échantillons superficiels de sol mouillés, test d'écrasement à sec). Les types d'érosion et l'activité biologique du sol sont notés par simple observation visuelle. Les indices de présence anthropique et l'estimation des différentes strates sont également jugés à l'œil nu. 


\section{Traitement des données}

Certains attributs vitaux de l'écosystème axés sur la diversité végétale (Aronson et al., 1995) sont intégrés dans l'analyse. Le logiciel Excel 2007 de Microsoft Office a été utilisé. L'application du test de khi-deux $\left(\mathrm{X}^{2}\right)$, notamment le test d'homogénéité, a été possible d'une part, en considérant l'équiprobabilité des différentes modalités et d'autre part, en négligeant les valeurs réelles obtenues inferieures à 5 (cas des types phytogéographiques et de l'état des espèces).

La réalisation du profil écologique de la végétation s'est faite en utilisant des paramètres de quantification des taxa (abondance, dominance, surface terrière, fréquence) tels que repris par Le Floc'h (2007). Ces mesures ont été complétées par l'évaluation de la densité des individus qui correspond au nombre de tiges par unité de surface.

L'analyse des résultats correspondant à la richesse spécifique s'est fondée sur des critères proposés par Daget et Poissonet (1991, 1997), Daget (2002), cités par Le Floc'h (2007). L'évaluation de la diversité floristique du parc s'est faite en utilisant les indices de diversité de Shannon-Weaver (H') et de Simpson (D), ou d'équitabilité de Piélou (E) cités par Sonké (1998), Ntoupka (1999), Le Floc'h (2007), Marcon (2011).

L'hétérogénéité du massif est évaluée à partir du coefficient de similitude floristique de Jaccard $(1902,1928)$, associé à la distance de Hamming $(\mathrm{H})$ proposée par Daget et al. (2003), cités par Le Floc'h (2007).

Par ailleurs, pour un taux de sondage de $1 \%$ (correspondant à une précision $\mathrm{P}_{0}=4 \%$ ), considéré comme acceptable pour un aménagement sylvo-pastoral par Arbonnier (1990) cité par Bellefontaine et al. (1997), il a été estimé le nombre $\mathrm{n}$ de placettes à prévoir tel que: $\mathrm{n} \geq \frac{\mathbf{4 ( \mathbf { C V } ) ^ { 2 }}}{\mathbf{P o}^{2}}, \mathrm{cv}=\frac{\sigma}{\overline{\mathrm{x}}}$ pour un paramètre donné, où $\quad \bar{x} \quad$ est la moyenne des valeurs obtenues dans les échantillons et $\sigma$ son écart type.

La description de la structure verticale de l'écosystème a été réalisée sur la base des paramètres tels que : la détermination de la proportion des différents types de tiges (tiges de régénération, tiges d'avenir, tiges moyennes, hautes tiges), la représentativité des espèces sarmenteuses ou buissonnantes, afin d'apprécier l'accessibilité du site et la répartition des différents types végétatifs. Parmi les différents types de tiges, les tiges de régénération ont été considérées comme ayant une taille inférieure ou égale à $1 \mathrm{~m}$; les tiges d'avenir celles dont la hauteur est comprise entre $] 1 \mathrm{~m} ; 1,5 \mathrm{~m}]$; les tiges moyennes situées dans l'intervalle ]1,5 m;3 m] et les hautes tiges se situant au-delà de $3 \mathrm{~m}$. La corrélation entre les circonférences et les hauteurs a aussi servi à l'analyse de cette structure verticale.

Une évaluation de l'aire de la distribution des différentes espèces a permis d'apprécier le taux d'endémisme sahélosoudanien. Les types phytogéographiques distingués sont définis par Letouzey (1985) ou Arbonnier (2004). Les espèces peuvent être pantropicales ou appartenir aux secteurs suivants: saharien (saha), saharo-sahélien (saha-sahé), sahélien (sahé), sahélo-soudanien (sahé-sou), soudano-sahélien (sou-sahé), soudanien (sou), soudano-guinéen (sou-gui) et guinéen (gui).

Les types végétatifs sont classés en 3 grands groupes, en fonction de la classification d'Arbonnier (2004): arbres, arbustes, lianes. Pour les espèces correspondant à plusieurs types végétatifs, l'état d'observation sur le terrain est privilégié. Les espèces sarmenteuses sont classées parmi les arbustes. 

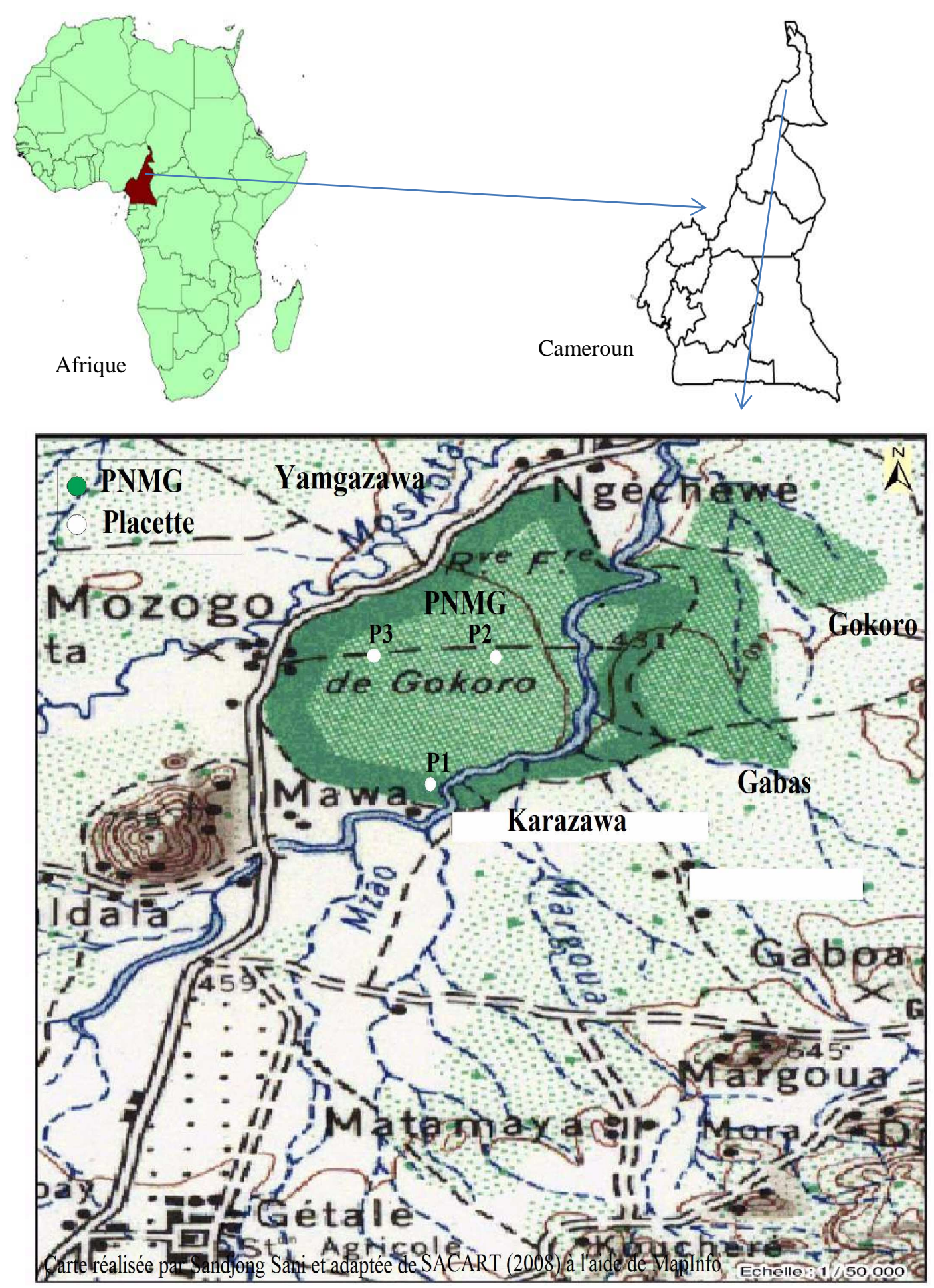

Figure 1: Carte représentant le PNMG avec localisation des placettes (Adaptation de la carte de SACART, 2008). 


\section{RESULTATS}

\section{Description simple du sol et physionomie de la végétation}

Deux types de texture ont été établis à l'issue du pré-inventaire: texture sablolimoneuse à $75 \%$ et texture limono-sableuse à $25 \%$. Le phénomène d'érosion est absent dans toutes les placettes d'inventaire. L'activité biologique du sol a été identifiée par la présence de fourmilières, de termitières cathédrales, de trous d'animaux fouisseurs tels que les reptiles et rongeurs (python et varans, rats,...) et plusieurs invertébrés en déplacement.

L'appréciation visuelle des différentes strates dans toutes les placettes donne une physionomie de forêts sèches claires à denses, avec parfois, une végétation ligneuse importante, à couvert fermé, et de multiples individus à hauteur supérieure à $15 \mathrm{~m}$. Le tapis graminéen associé à d'autres herbacées observées çà et là, se trouve très dense par endroit où l'accès de l'Homme est perceptible (défrichement et coupe de bois). Ces observations attestent qu'à certains endroits anthropisés ou affectés, la capacité de résilience est gardée.

\section{Richesse et fréquence spécifiques en ligneux \\ L'analyse de la richesse floristique a} permis d'identifier 62 espèces dans l'ensemble des placettes. Certaines placettes se caractérisent par une richesse spécifique plus élevée que d'autres (51 espèces dans la placette $\mathrm{P} 1,40$ espèces dans la placette $\mathrm{P} 2$, 37 espèces dans la placette P3). Le Tableau 1 donne la liste des espèces végétales, les noms vernaculaires, les familles correspondantes. Il y a une gradation descendante du nombre d'espèces de la placette P1 à la placette P3. L'influence du rivage du Mayo ou du basfond est déterminante pour cette répartition d'espèces. La flore du parc est très riche, avec 62 espèces et 26 familles présentes. Les espèces les plus fréquentes $(100 \%$ de fréquence spécifique centésimale) se trouvant dans l'ensemble des placettes sont: Abrus precatorios, Acacia ataxacantha, Acacia senegal, Anogeissus leiocarpus, Balanites aegyptiaca, Boscia senegalensis, Capparis fascicularis, Celtis integrifolia, Grewia bicolor, Stereospermum kunthianum, Opilia celtidifolia, Hexalobus monopetalus, Ziziphus spina-christi, Grewia barteri, Commiphora pedunculata, Dalbergia melanoxylon, et Tamarindus indica.

\section{Homogénéité ou hétérogénéité du massif}

En calculant la distance de Hamming pour l'évaluation de l'homogénéité ou l'hétérogénéité du massif, il est noté une différence floristique forte entre $\mathrm{P} 2$ et $\mathrm{P} 3$ (64\%), moyenne entre P1 et P3 $(45,61 \%)$ et P1 et P2 $(42,18 \%)$. Dans la placette P2, située dans un site occupé dans le passé par un ancien village, la différence floristique n'est pas très forte avec les autres placettes. Il s'agit de l'atteinte d'un stade alternatif stable.

\section{Abondance et dominance des espèces}

Plusieurs espèces se distinguent par leur effectif élevé. Les espèces assez représentatives pour caractériser la végétation du parc sont : Acacia ataxacantha $(33,80 \%)$, Hexalobus monopetalus (16,66\%), Anogeissus leiocarpus (6,26\%), Grewia bicolor (4,96\%), Tamarindus indica $(4,20 \%)$, Balanites aegyptiaca (3,57\%), Diospyros mespiliformis (3,21\%), Grewia barteri (2,65\%), Boscia senegalensis (2,65\%), Opilia celtidifolia (2,38\%), Cissus quadrangularis $(2,26 \%)$, Celtis integrifolia (2,18\%), Piliogstima reticulatum (1,62\%), Dalbergia melanoxylon (1,26\%), Combretum fragrans $(1,19 \%)$, Capparis fascicularis $(0,91 \%)$, Stereospermum kunthianum $(0,79 \%)$, Cadaba farinosa $(0,79 \%)$. Plusieurs 
espèces peuvent être considérées comme accidentelles ou rares, car elles sont représentées par un seul individu: Securidaca longepedunculata, Acacia sp., Syzygium guineense, Combretum collinum, Holarrhena floribunda, Sterculia setigera, Acacia nilotica, Allophylus spicatus, Strychnos cocculoüdes, Commiphora africana, Combretum molle, Vitex doniana, Prosopis africana, ... D'autres espèces sont sousreprésentées, parce que liées à la présence humaine ou à leur probable introduction par l'avifaune ou les Chiroptères: Azadirachta indica, Adansonia digitata. Les espèces arborescentes sont les plus dominantes (Tableau 2). La présence d'un individu d'Adansonia digitata de $13,10 \mathrm{~m}$ de circonférence, soit $4,17 \mathrm{~m}$ de diamètre et 35 $\mathrm{m}$ de hauteur marque significativement la surface terrière totale $\left(134,55 \mathrm{~m}^{2} / \mathrm{ha}\right)$.

\section{Abondance et dominance des familles}

Sur les 2520 individus inventoriés, la famille des Mimosaceae est la plus abondante, avec 896 individus recensés. Elle est suivie respectivement par les familles Annonaceae, Combretaceae, Tiliaceae, Caesalpiniaceae, Capparaceae, Balanitaceae, Ebenaceae, Opiliaceae, Ampelidaceae et Ulmaceae. Les familles les moins représentées en individus ou rares sont les suivantes: Loganiaceae, Anarcadiaceae, Polygalaceae, Clusiaceae Myrtaceae, Lamniaceae, Sapindaceae, Sterculiaceae. Certaines familles, bien que importantes par leur surface terrière, ont une abondance moins significative. Il s'agit de Bombacaceae et Bignoniaceae.

\section{Densité des individus}

Avec une densité moyenne des trois placettes évaluée à 2972 tiges/hectare, il y a une assez bonne répartition des tiges dans le massif. Ceci prouve que l'aire protégée n'a pas subi de perturbations majeures (Tableau $3)$.

\section{Taux de sondage d'aménagement prévisionnel}

En prenant comme paramètres le nombre d'espèces et la densité, le nombre de placettes circulaires de $2826 \mathrm{~m}^{2}$ à prévoir pour un aménagement sylvo-pastoral devrait être supérieur ou égal à 53 pour la densité et à 74 pour la richesse spécifique.

\section{Diversité et équitabilité floristiques}

Les indices de diversité de ShannonWeaver ( $\left.H^{\prime}\right)$ calculés varient très peu d'une placette à l'autre. Ils traduisent la très grande richesse floristique du site d'étude. Le calcul de l'équitabilité de Piélou (E) rattachée à l'indice de Shannon confirme les valeurs d'indices de diversité élevées sachant que les valeurs qui se rapprochent de l'unité ont une diversité spécifique grande. Il y a donc $63 \%$ de chance qu'il y ait équilibre dans la répartition des espèces dans le parc. L'indice de Simpson (D) prouve que la probabilité (1D) pour que deux individus pris au hasard soient de différentes espèces est très élevée. Le Tableau 4 donne les valeurs des différents indices calculés.

\section{Structure verticale \\ Diversité des tiges et types végétatifs}

En observant la Figure 2, il est remarquable $\left(X^{2}>16,26\right.$; probabilité < $0,001)$ dans cette répartition non homogène, que les tiges les moins représentées sont les tiges dites d'avenir et les tiges de régénération, avec une différence très grande par rapport aux autres tiges (structure en L). La Figure 3 qui illustre le nombre d'individus correspondant à chaque type végétatif (arbres, arbustes, lianes), montre très significativement que les espèces arbustives sont les plus importantes $\left(X^{2}=1595,74\right.$ avec 
un risque presque nul). La proportion des individus sarmenteux formant des touffes est très élevée $(30 \%)$. Le sous-bois est rendu parfois inaccessible, surtout que l'espèce la plus concernée est Acacia ataxacantha.

\section{Corrélation circonférences / hauteurs}

La corrélation établie entre les circonférences et les hauteurs des individus montre un ajustement meilleur obtenu avec une courbe dont l'équation est celle d'une fonction puissance, avec un coefficient de corrélation $\mathrm{R}^{2}=0,217$ (Figure 4).

\section{Distribution phytogéographique}

Les préférences phytogéographiques des espèces s'étendent de la zone saharosahélienne à la zone guinéenne. Dans cette distribution, les ligneux sahéliens à soudaniens ont le plus important nombre d'individus et sont considérés comme espèces clés de voute $\left(X^{2}>34,52\right.$; probabilité $<0,001)$. Ils sont suivis par les ligneux inféodés aux zones soudaniennes et guinéennes. Dix-sept types phytogéographiques ont été attribués, témoignant toujours de la grande diversité végétale du parc (Figure 5). Il y a une forte migration d'éléments spécifiques des domaines soudanien et guinéen.

\section{Perturbations de l'équilibre biologique: degré d'anthropisation}

Les coupes de bois, les pistes braconnières, les prélèvements de pailles, des creux d'igname, sont observés dans toutes nos placettes. La Figure 6 montre que les ligneux coupés en têtard, émondés ou étêtés sont les plus nombreux $\left(\mathrm{X}^{2}>20,51\right.$ et risque d'uniformité des différents états presque nul). Les espèces les plus convoitées (étêtées, coupées en têtard ou émondées) sont:
Anogeissus leiocarpus, Dalbergia melanoxylon, Acacia ataxacantha, Balanites aegyptiaca, Tamarindus indica. Le taux de mortalité élevé de certaines espèces (Anogeissus leiocarpus, Acacia ataxancantha, Grewia bicolor, Stereospermum kunthianum...) et le port tortueux ou brisé d'autres espèces (Holarrhena floribunda, Anogeissus leiocarpus, Grewia bicolor, Stereospermum kunthianum, Piliogstima reticulatum, Piliogstima thonningii, Hexalobus monopetalus, Combretum aculeatum, Combretum nigricans, ...) expriment le dynamisme naturel de compétition interspécifique.

Déterminisme de la conservation : indices d'appartenance à l'écosystème de référence

Quelques indices identifiés, après analyse des données, peuvent conférer au PNMG les attributs d'un écosystème de référence en zone sahélo-soudanienne pour justifier sa protection intégrale. Sa diversité compositionnelle est particulièrement élevée avec une flore riche constituant une banque de gènes. L'abondance et la dominance des familles et des espèces montrent sa très grande hétérogénéité. La densité et la surface terrière des individus sont également élevées, signes d'une faible pression anthropique. Il est noté aussi la forte présence d'éléments spécifiques soudaniens ou guinéens, les fortes proportions d'individus sarmenteux et lianescents, rendant parfois le sous-bois impénétrable, et des mécanismes d'auto destruction indiquant que cet écosystème a emprunté une trajectoire évoluant vers une végétation sahélo-soudanienne typique sans grandes perturbations. 
R. C. SANDJONG SANI et al. / Int. J. Biol. Chem. Sci. 7(6): 2434-2449, 2013

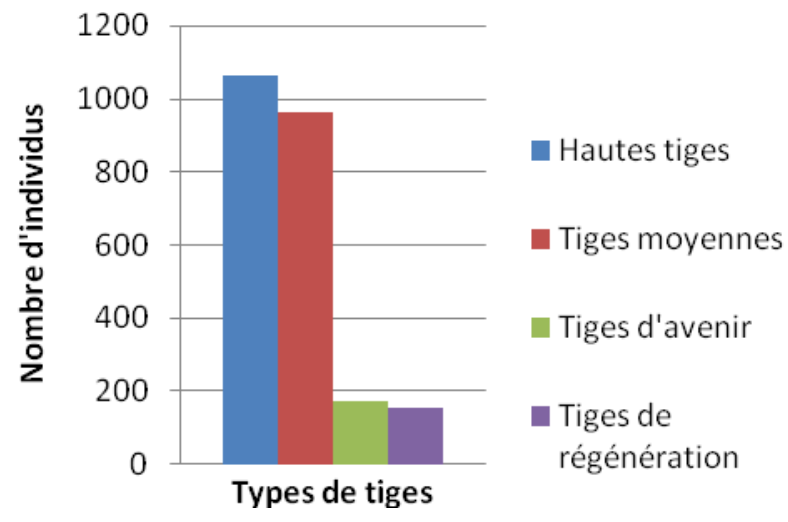

Figure 2: Abondance des différents types de tiges.

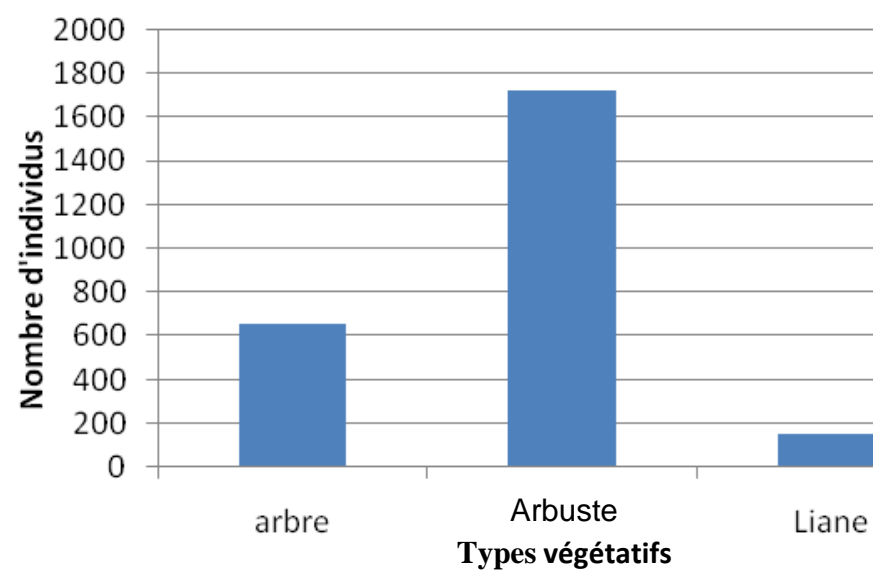

Figure 3: Représentativité des types végétatifs de ligneux.

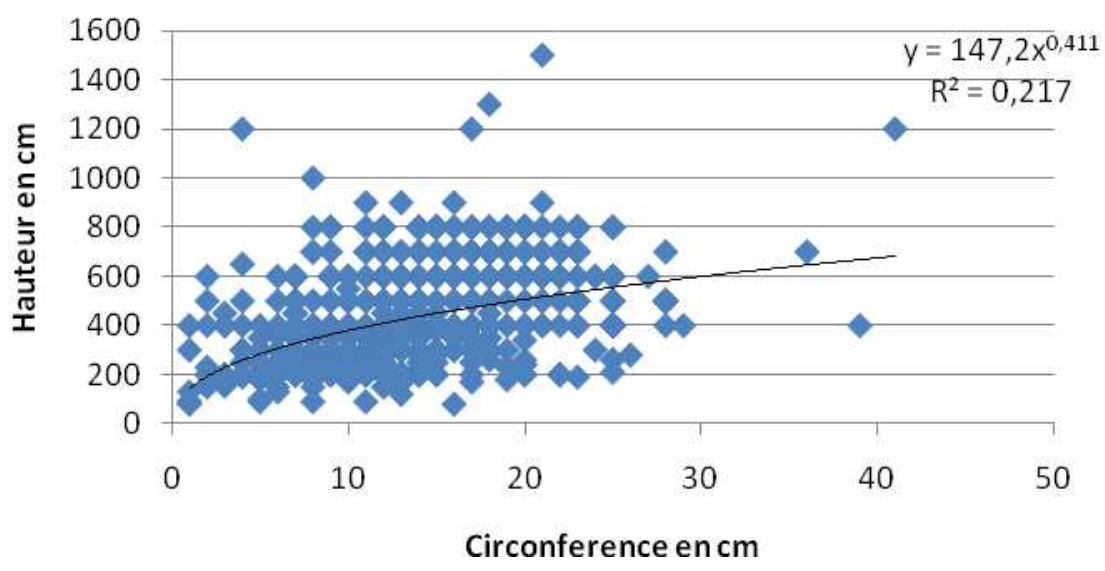

Figure 4: Courbe de corrélation circonférence/hauteur. 


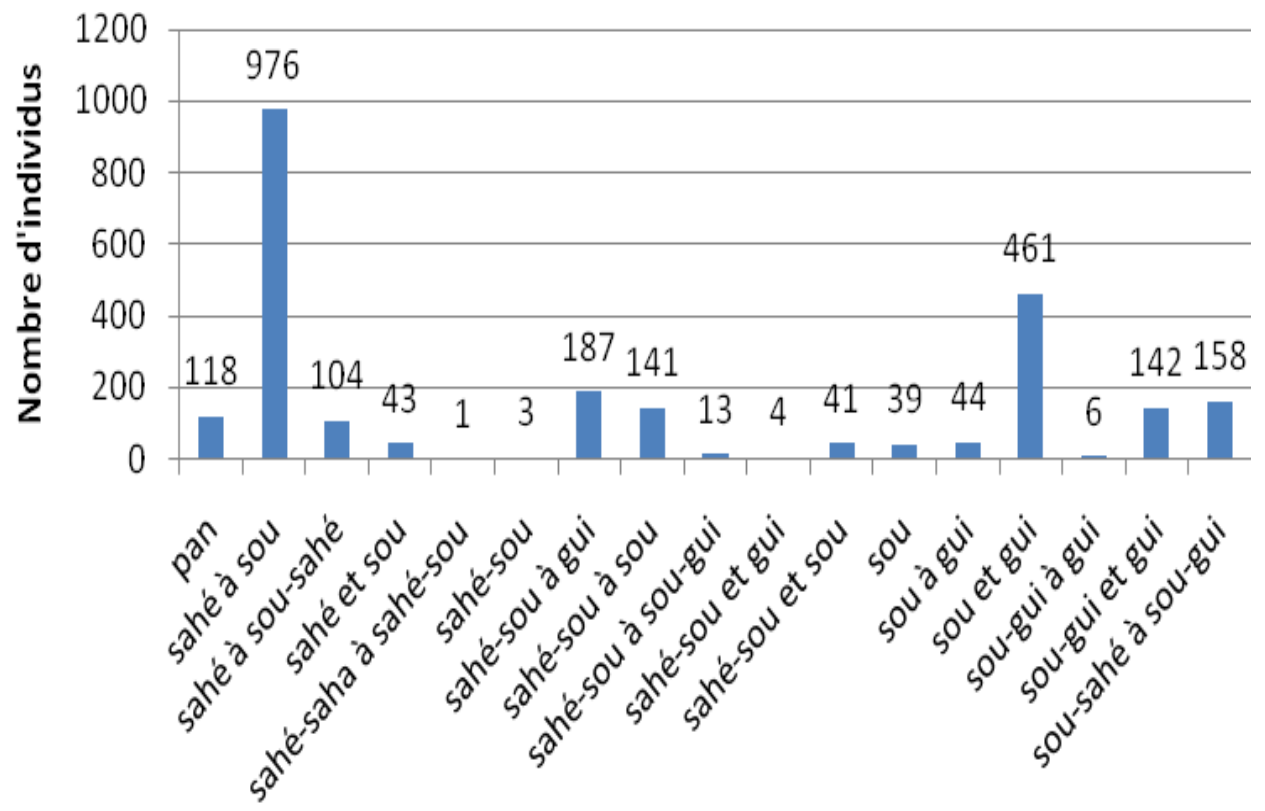

Types phytogéographiques

Figure 5: Types phytogéographiques des espèces. Gui= Guinéen; Sahé = sahélien; Sou = soudanien; Sahé-sou = sahélo-soudanien; Sou-gui $=$ soudano-guinéen; Sou-sahé = soudano-sahélien; saha-sahé $=$ saharo-sahélien.

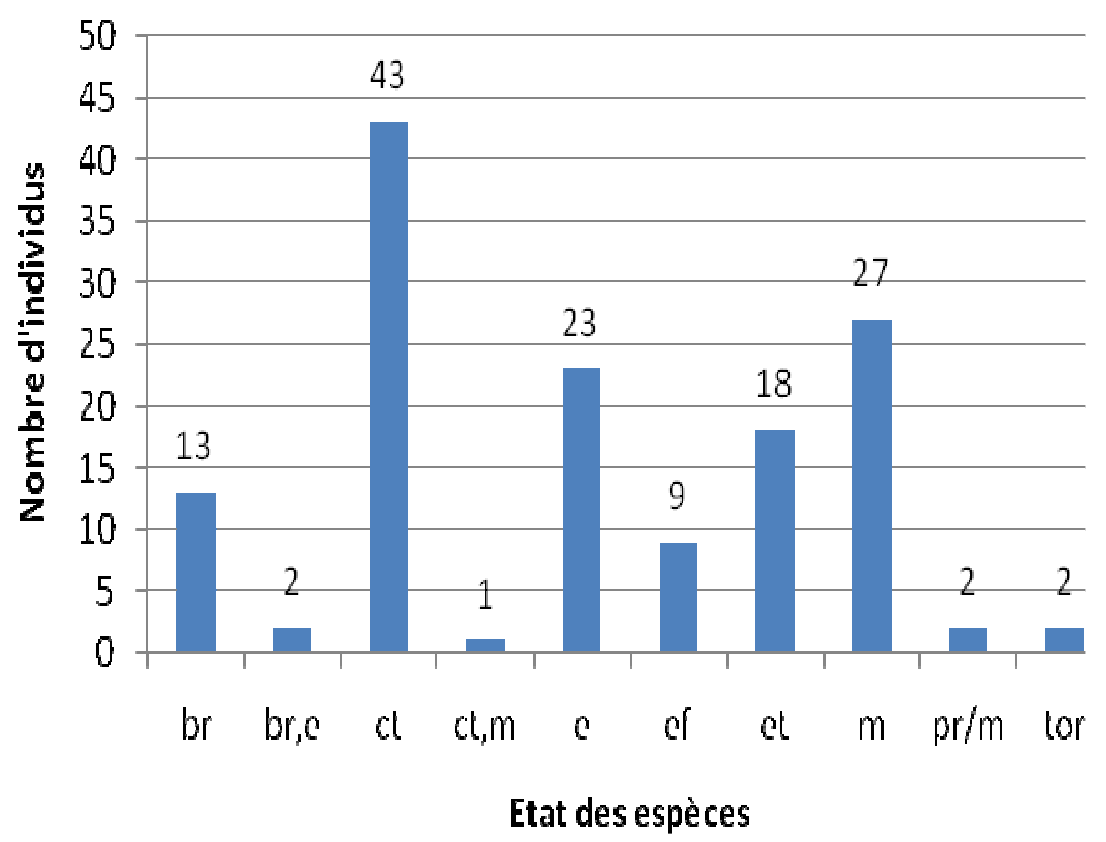

Figure 6: Etat des différents individus recensés. br= brisé; e= émondé ; ct= coupé en têtard ; ef= effeuillé; $\mathrm{m}=$ mort; tor=tortueux; et=étêté; $\mathrm{pr} / \mathrm{m}=$ presque mort. 
Tableau 1: Liste des espèces végétales: noms scientifiques, noms vernaculaires et familles.

\begin{tabular}{|c|c|c|c|}
\hline $\mathbf{N}^{\mathbf{O}}$ & Noms scientifiques & Noms vernaculaires & Familles \\
\hline 1 & Abrus precatorius $\mathrm{L}$. & Ouroumtekire (Md) & Mimosaceae \\
\hline 2 & Acacia ataxacantha DC. & Zaza (Md) & Mimosaceae \\
\hline 3 & Acacia nilotica Willd. & Kangar (Mf) & Mimosaceae \\
\hline 4 & $\begin{array}{l}\text { Acacia polyacantha subsp. campylacantha } \\
\text { (Hochst.) Roberty }\end{array}$ & Patouki Danehi $(\mathrm{Fu})$ & Mimosaceae \\
\hline 5 & Acacia sieberiana DC. & Goloumb (Mf) & Mimosaceae \\
\hline 6 & Acacia senegal (L.) Willd. & Zoma Dou Eh (Md) & Mimosaceae \\
\hline 7 & Acacia sp. & Napzehe (Md) & Mimosaceae \\
\hline 8 & Adansonia digitata (Linn) & Boki (Fu) & Bombacaceae \\
\hline 9 & Allophylus spicatus (Poir.) Radlk. & Oufwayam (Mf) & Sapindaceae \\
\hline 10 & Ancylobotrys amoena Hua & Delbi Gooko (Fu) & Apocynaceae \\
\hline 11 & Anogeissus leiocarpus (DC.) Guill. \&Perr. & Daoui (Mf) & Combretaceae \\
\hline 12 & Azadirachta indica A. Juss. & Ngagné (Mf) & Meliaceae \\
\hline 13 & Balanites aegyptiaca $($ L.) Del. & Ltaram (Mf) & Balanitaceae \\
\hline 14 & Bombax costatum Pellegr. \&Vuil. & Bokoum (Mf) & Bombacaceae \\
\hline 15 & Boscia senegalensis (Pers.) Lam. & Tambila (Md) & Capparaceae \\
\hline 16 & Boswellia dalzielii Hutch. & Babele (Mf) & Burseraceae \\
\hline 17 & Cadaba farinosa Forssk. & Ardjekwa (Md) & Capparaceae \\
\hline 18 & Caparis fascicularis DC. & Brouglo Brouglo (Mf) & Capparaceae \\
\hline 19 & Capparis sp. & Nogobala (Md) & Capparaceae \\
\hline 20 & Carissa edulis (Forssk.) Vahl & Bodroum (Mf) & Apocynaceae \\
\hline 21 & Celtis integrifolia Lam. & Chechébé (Mf) & Ulmaceae \\
\hline 22 & Cissus quadrangularis $\mathrm{L}$. & Madzaf-Zi (Mf) & Ampelidaceae \\
\hline 23 & $\begin{array}{l}\text { Clerodendrum capitatum (Willd.) Schum. } \\
\text { \&Thonn. }\end{array}$ & Tchitimagoulva (Md) & Verbenaceae \\
\hline 24 & Combretum aculeatum Vent. & Gourdiak (Mf) & Combretaceae \\
\hline 25 & Combretum collinum Fres. & Uhler (Mf) & Combretaceae \\
\hline 26 & Combretum fragrans $\mathrm{F}$. Hoffm. & Guijibet (Mf) & Combretaceae \\
\hline 27 & Combretum molle R.Br. ex G.Don. & DoodjiBodehi $(\mathrm{Fu})$ & Combretaceae \\
\hline 28 & Combretum nigricans Lepr. & Verchengue (Md) & Combretaceae \\
\hline 29 & Commiphora africana (A. Rich.) & Namcheke (Md) & Burseraceae \\
\hline 30 & $\begin{array}{l}\text { Commiphora pedunculata (Kortschy \& Peyr.) } \\
\text { Engl }\end{array}$ & Demteuk (Mf) & Burseraceae \\
\hline 31 & Dalbergia melanoxylon Guill. \& Perr. & Dayik (Mf) & Fabaceae \\
\hline 32 & Dichrostachys cinerea (L.) Wight \& Arn. & Kekirmbe (Mf) & Mimosaceae \\
\hline 33 & Diospyros mespiliformis Hochst. & Hawoun (Mf) & Ebenaceae \\
\hline 34 & Entada abyssinica Steud. ex A. Rich. & Yeyeh (Md) & Mimosaceae \\
\hline 35 & Grewia barteri Burret & Schekur (Mf) & Tiliaceae \\
\hline 36 & Grewia bicolor Juss. & Djere (Md) & Tiliaceae \\
\hline 37 & Grewia flavescens Juss. & Lalaopa (Md) & Tiliaceae \\
\hline 38 & Guieria senegalensis Gmel. & Jewoud (Mf) & Combretaceae \\
\hline 39 & Hexalobus monopelatus (A.Rich.) Engl.\&Diels & Koulha (Md) & Annonaceae \\
\hline 40 & $\begin{array}{l}\text { Holarrhena floribunda (G.Don) Th.Dur. } \\
\& \text { Schinz }\end{array}$ & Safa $(\mathrm{Md})$ & Apocynaceae \\
\hline 41 & Khaya senegalensis (Desr.) A.Juss. & Serehi $(\mathrm{Fu})$ & Meliaceae \\
\hline 42 & Lonchocarpus laxiflorus Guill. \& Perrott & Vava (Mf) & Fabaceae \\
\hline 43 & Maerua crassifolia Forssk. & Hatsoanoudje (Md) & Capparaceae \\
\hline 44 & Maerua oblongifolia (Forssk.) A.Rich. & Kedak (Mf) & Capparaceae \\
\hline 45 & $\begin{array}{l}\text { Opilia celtidifolia (Guill. \& Perr.) Walp. Endl. ex } \\
\text { Walp. }\end{array}$ & DelbiDeboh (Fu) & Opiliaceae \\
\hline
\end{tabular}




\begin{tabular}{|c|c|c|c|}
\hline 46 & Piliostigma reticulatum(DC.) Hochst. & Wouma (Mf) & Caesalpiniaceae \\
\hline 47 & Piliostigma thonningii(Schum.) Milne-Redh. & Wutouf (Mf) & Caesalpiniaceae \\
\hline 48 & Prosopis africana (Guill. \&Perr.) Taub. & Tsakitsak (Mf) & Mimosaceae \\
\hline 49 & Psorospermum sp. & Azachzara (Md) & Clusiaceae \\
\hline 50 & Pteleopsis suberosa Engl. \&Diels & Nader (Mf) & Combretaceae \\
\hline 51 & Pterocarpus erinaceus Poir. & $\operatorname{Dozouk}(\mathrm{Mf})$ & Fabaceae \\
\hline 52 & Sclerocarya birrea (A. Rich.) Hochst & Malolea (Md) & Anarcadiaceae \\
\hline 53 & Securidaca longepedunculata Fres. & Alali $(\mathrm{Fu})$ & Polygalaceae \\
\hline 54 & Sterculia setigera Del. & ZhawaZehe (Md) & Sterculiaceae \\
\hline 55 & Stereospermum kunthianum Cham. & Kulbita (Md) & Bignoniaceae \\
\hline 56 & Strychnos cocculoides Baker. & Sef-Zehe (Md) & Loganiaceae \\
\hline 57 & Strychnos spinosa Lam. & Katagredeuk (Mf) & Loganiaceae \\
\hline 58 & Syzygium guineense (Willd.) & ZhadaouiHude (Md) & Myrtaceae \\
\hline 59 & Tamarindus indica Linné & Broum (Mf) & Caesalpiniaceae \\
\hline 60 & Vitex doniana Sweet. & Zkad (Mf) & Lamniaceae \\
\hline 61 & Ziziphus mucronata Willd. & Vrukaza (Mf) & Rhamnaceae \\
\hline 62 & Ziziphus spina-christi (L.) Desf. & Wandre (Mf) & Rhamnaceae \\
\hline
\end{tabular}

Tableau 2: Surfaces terrières et dominances de quelques espèces par ordre décroissant.

\begin{tabular}{lccc}
\hline Espèces & $\begin{array}{c}\text { Surfaces terrières } \\
\left(\mathbf{m}^{2} / \mathbf{h a}\right)\end{array}$ & $\begin{array}{c}\text { Dominances } \\
\text { relatives }\end{array}$ & $\begin{array}{c}\text { Dominances } \\
\text { absolues }\end{array}$ \\
\hline Adansonia digitata & 48,39 & 0,35 & 35,96 \\
Tamarindus indica & 18,19 & 0,13 & 13,51 \\
Anogeissus leiocarpus & 13,57 & 0,10 & 10,08 \\
Balanites aegyptiaca & 9,24 & 0,068 & 6,872 \\
Acacia ataxacantha & 6,03 & 0,044 & 4,48 \\
Stereospermum kunthianum & 3,56 & 0,026 & 2,64 \\
Piliogstima thonningii & 3,32 & 0,024 & 2,47 \\
Bombax costatum & 3,022 & 0,022 & 2,25 \\
Combretum fragrans & 3,011 & 0,022 & 2,24 \\
Acacia sieberana & 3,010 & 0,022 & 2,23 \\
Celtis integrifolia & 2,81 & 0,020 & 2,09 \\
Sclerocarya birrea & 2,57 & 0,019 & 1,91 \\
Hexalobus monopetalus & 2,22 & 0,016 & 1,65 \\
Pterocarpus erinaceus & 1,89 & 0,014 & 1,40 \\
Piliogstima reticulatum & 1,63 & 0,012 & 1,21 \\
Diospyros mespiliformis & 1,50 & 0,011 & 1,12 \\
Combretum nigricans & 1,44 & 0,010 & 1,07 \\
Dalbergia melanoxylon & 1,22 & 0,0091 & 0,91 \\
Ziziphus spina-christi & 1,16 & 0,0086 & 0,86 \\
Lonchocarpus laxiflorus & 0,78 & 0,0058 & 0,58 \\
Total partiel & 128,56 & 0,933 & 95,53 \\
\hline
\end{tabular}


Tableau 3: Densité des individus.

\begin{tabular}{lcc}
\hline Placette & $\begin{array}{c}\text { Densité (nombre de tiges } \\
\text { par hectare) }\end{array}$ & $\begin{array}{c}\text { Densité moyenne (nombre } \\
\text { de tiges par hectare) }\end{array}$ \\
\hline P1 & 3036 & 2972 \\
P2 & 2498 & \\
P3 & 3342 & \\
\hline
\end{tabular}

Tableau 4 : Valeurs des différents indices de diversité calculés.

\begin{tabular}{ccccc}
\hline Indices & \multicolumn{3}{c}{ Placettes } & Global \\
\cline { 2 - 4 } & P1 & P2 & P3 & \\
\hline H' (bit) & 3,8 & 3,40 & 3,41 & 3,75 \\
E & 0.67 & 0,63 & 0.65 & 0,63 \\
D & 0,14 & 0,18 & 0,16 & 0,15 \\
1-D & 0.86 & 0,86 & 0.83 & 0,84 \\
\hline
\end{tabular}

\section{DISCUSSION}

\section{Echantillonnage et analyse de l'état de l'horizon superficiel du sol}

La relative hétérogénéité entre les placettes montre qu'il faut envisager un inventaire avec un taux de sondage plus grand pour obtenir des caractéristiques plus précises du parc. Toutefois, selon Marcon (2011), l'augmentation de la surface d'échantillonnage n'est pas toujours une solution adaptée en zone tropicale, puisqu'elle se solde toujours par l'augmentation du nombre d'espèces. L'accessibilité du site, évaluée ici par le taux d'espèces sarmenteuses (30\%), sera déterminante pour une prospection d'aménagement plus importante.

La prépondérance de la texture sablolimoneuse dans l'horizon superficiel, l'absence d'érosion et l'activité biologique présentes dans le sol sont des caractéristiques pédologiques favorables à l'épanouissement de la végétation car, la capacité de rétention d'eau et d'air est relativement élevée, l'infiltration, la circulation de l'eau et la minéralisation facilitées. A partir de ces données préliminaires, le site d'étude ne présente presque pas de symptômes visibles de dégradation des terres. D'après Toutain et al. (2006) cités par Conedera et al. (2010), ces indices sont: la raréfaction, voire la disparition de la flore herbacée et arborée, la réduction de la taille des arbres qui résistent, la réduction de l'activité biologique du sol et l'augmentation des phénomènes de ruissellement superficiel et d'érosion.

\section{Diversités compositionnelle et structurale}

Les attributs vitaux (Aronson et al., 1995) sont importants pour le suivi écologique, c'est-à-dire une activité qui consiste à collecter des informations sur le milieu, sur sa biodiversité dans le temps et dans l'espace, en vue de mieux le connaître et de contribuer efficacement à sa gestion (Clarke, 1980; Donfack, 2009). Ntoupka (1999) obtient, dans un écosystème de savane naturelle, soumis à diverses perturbations anthropiques en zone soudano-sahélienne au Nord-Cameroun (Laf), une richesse floristique totale de 45 espèces de ligneux, des valeurs maximales d'indices de diversité de Shannon variant de 3,1 à 4,5 bits et d'équitabilité de 
0,60 à 0,78 . Les différences constatées soutiennent seulement la grande richesse en ligneux du parc (62espèces). Afin de mieux appréhender ces caractéristiques floristiques, d'autres données sont comparées à celles présentées par Hountondji (2008) relatives à plusieurs zones éco-géographiques au Benin et par Adjonou et al. (2009) dans les forêts claires à Anogeissus leiocarpus du Parc national Oti-Ke'ran au Nord-Togo. En sousestimant les différences dans les méthodes d'inventaire, il ressort de l'analyse un rapprochement de la végétation du parc à celles des zones phytogéographiques beaucoup plus humides (Sud-Soudanienne et Nord-Soudanienne). Les valeurs de surface terrière, de densité, et de richesse spécifique obtenues au parc sont parfois nettement supérieures, même quand il s'agit de forêt claire ou de forêt dense sèche. De plus, dans la réserve de Ngazobil au Sénégal (27ha), réputée comme vestige de l'écosystème forestier de la zone sahélo-soudanienne de la «petite côte» dans ce pays, Diatta et al. (2009) recensent 60 espèces réparties en 47 genres et 25 familles. Le peuplement des ligneux de cette végétation se caractérise par l'indice de Shannon évalué à 1,87 bit, une densité observée de 1464 individus/ha et une surface terrière de $59,6 \mathrm{~m}^{2} /$ ha. Ces résultats corroborent majoritairement les atouts floristiques exceptionnels conférés au PNMG. Marcon (2011) considère toutefois que la richesse spécifique, comme indice de diversité, pose le problème dans son interprétation de la surface d'échantillonnage.

Par ailleurs, la structure en L illustrée par la végétation a été également observée en zone soudanienne à Ngaoundéré au Cameroun et considérée comme un indice de dégradation (Tchobsala et al., 2010). La prépondérance des individus de taille moyenne ou haute est une caractéristique de la maturité de cet écosystème inclus dans une zone où la trajectoire des peuplements aboutit généralement à la domination d'arbustes (steppes ou savanes arbustives) correspondants aux espèces sahélo- soudaniennes. L'irradiation d'espèces saharosindiennes en région soudano-zambézienne au Mali étaye les modifications bioclimatiques et suggère un réexamen des distributions floristiques (Habiyaremye et Roche, 2004). La parenté tropicale des climats sahéliens et soudaniens (Hiernaux et Le Houérou, 2006) justifie la prédominance d'espèces ligneuses ayant une affinité aux domaines correspondants.

Se référant à l'information donnée par Ntoupka (1999), la grande accumulation de paille au sol serait à l'origine d'une diminution des possibilités de germination et donc des tiges de régénération et d'avenir en l'absence de feux. L'abondance élevée d'Acacia ataxacantha peut se justifier par l'absence de feux car, selon Ntoupka (1999), les feux empêchent la formation des buissons dans les pâturages en tuant les arbres et les arbustes buissonnants à l'exemple d'Acacia ataxacantha. Ces observations reflètent la grande préservation du site d'étude et ses attributs d'écosystème de référence. De la corrélation circonférence /hauteur, la Figure 6 montre que les hauteurs et les circonférences d'un grand nombre d'individus ne sont pas en cohérence. La concurrence pour la lumière serait à l'origine de ces décalages.

\section{Impact des perturbations anthropiques}

Les indices de présence anthropique dans le parc ont été relevés au cours de notre pré-inventaire pour apporter un plus aux résultats de l'enquête sur la gestion du parc effectué concomitamment auprès de la population riveraine (Sandjong, 2012). Ils sont susceptibles de perturber les caractéristiques écologiques évaluées. Le taux de mortalité $(1,19 \%)$ des individus n'est pas très élevé. Dans les forêts claires à Anogeissus leiocarpus du Parc National Oti-Ke'ran au Nord-Togo, les changements climatiques se traduisent par une mortalité anormalement accrue des arbres (taux de mortalité voisin de $21,2 \%$ ), surtout au niveau des individus de petits diamètres (Adjonou et al., 2009). 


\section{Conclusion}

A partir d'un pré-inventaire floristique des ligneux et d'un essai d'analyse de l'état de l'horizon de surface du sol, le PNMG a dévoilé plusieurs caractéristiques lui conférant un statut d'écosystème de référence en zone sahélo-soudanienne. Les diversités compositionnelle et structurale illustrent sa grande préservation, malgré la mise en évidence de l'action destructrice de l'Homme par quelques données obtenues. Une étude écologique plus approfondie, se fondant sur ce travail préliminaire favoriserait son réel suivi et son aménagement. L'accent pourrait être mis sur le dynamisme spatio-temporel des espèces et les scenarii de prévision de sa trajectoire.

\section{REMERCIEMENTS.}

Ce travail a été réalisé avec l'accord institutionnel de l'Ecole Normale Supérieure de l'Université de Maroua au Cameroun. Nous exprimons notre grande reconnaissance à cette institution universitaire. Notre gratitude s'oriente aussi vers toutes les personnes qui, par leur disponibilité ou leur participation, ont facilité le bon déroulement du pré-inventaire et des analyses.

\section{REFERENCES}

Adjonou K, Bellefontaine R, Kokou K. 2009. Les forêts claires à Anogeissus leiocarpus du Parc national Oti-Ke'ran au Nord-Togo : structure, dynamique et impacts des modifications climatiques. Sécheresse, 20(4): 394-6.

Arbonnier M. 2004. Arbres, Arbustes et Lianes des Zones Sèches d'Afrique de l'Ouest (4ème édn). CIRADMARGRAF-MNHN : Montpellier France ; 573 p.

Aronson J, Floret C, Le Floc'h E, Ovalle C, Pontanier R. 1995. Restauration et réhabilitation des écosystèmes dégradés en zone aride et semi aride, le vocabulaire et les concepts. In L'Homme Peut-il Refaire ce qu'il a Défait? Pontanier, M'Hiri, Aronson, Akrimi, Le
Floc'h (éds). John Libbey Eurotext : Montrouge, France ; 11-29.

Bellefontaine R, Gaston A, Petrucci Y. 1997. Aménagement des forêts tropicales sèches. FAO, p. 1-50.

Brabant P, Gavaud M. 1985. Les sols et les Ressources en Terres du Nord Cameroun: Cartes et Notice Explicative, ORSTOM : Paris ; p19.

Clarke R. 1980. The Handbook of Ecological Monitoring. GEMS/UNEP publication, Press Oxford: UK; 1-30.

Diatta CD, Gueye M, Koma S, Akpo LE. 2009. Diversité de la flore et de la végétation ligneuse de la réserve Ngazobil (Joal-Fadiouth) au Sénégal. $J$. Sc., 9(3):1-13.

Djoufack-Manetsa V. 2011. Étude multiéchelles des précipitations et du couvert végétal au Cameroun : Analyses spatiales, tendances temporelles, facteurs climatiques et anthropiques de variabilité du NDVI. Thèse de Doctorat, Université de Yaoundé I, Université de Bourgogne, 303 p.

Donfack P. 2009. Outils nécessaires à la mise en œuvre d'un système de suivi écologique pour les aires protégées du Cameroun, National Information Track, MINFOF, $143 \mathrm{p}$.

Donfack P, Seiny Boukar L, M'Biandoun M. 1996. Les caractéristiques du milieu physique: Actes de l'atelier d'échanges sur le thème : Agriculture des savanes du Nord Cameroun CIRAD, p. 29 - 42.

FAO. 2010. Lignes directrices pour la gestion durable des forêts en zones arides d'Afrique subsaharienne, Document de travail sur les Forêts et la Foresterie en zones arides, $\mathrm{n}^{\circ} 1,68 \mathrm{p}$.

Habiyaremye FX, Roche E. 2004. Contribution à l'observation de variations environnementales au Mali par l'analyse d'un transect phytogéographique Bamako- Tombouctou. Geo-Eco-Trop., 28: 1-14.

Hiernaux P, Le Houérou HN. 2006. Les parcours du Sahel. Sécheresse, 17(1-2): 51-71.

Hountondji HY. 2008. Dynamique environnementale en zones sahélienne et soudanienne de l'Afrique de l'Ouest : Analyse des modifications et évaluation 
de la dégradation du couvert végétal, Thèse de Doctorat, Université de Liège, Académie Universitaire WallonieEurope, Belgique, 153p.

Le Floc'h E. 2007. Guide ROSELT/OSS pour l'Etude et le Suivi de la Flore et la Végétation. Collection ROSELT/OSS, CT $\mathrm{n}^{\circ} 1$ : Tunis ; $175 \mathrm{p}$.

L'Hote Y. 1998. Climatologie et AgroClimatologie de la Province de l'Extrême-Nord (P.E.N) au Cameroun, Préparation de l'Atlas de la P.E.N. Editeur Scientifique Christian Seignobos, ORSTOM : Paris ; 31 p.

Letouzey R. 1985. Notice de la Carte Phytogéographique du Cameroun, Domaine Sahélien et Domaine Soudanien. IRA : Toulouse; 1-26.

Marcon E. 2011. Mesures de la Biodiversité, Ecologie des Forêts de Guyane. CNRS, CIRAD, INRA: Paris; 42 p.

M'biandoun M, Guibert H, Olina JP. 2002. 2002. Caractérisation du climat dans quatre terroirs de la zone soudanosahélienne du Nord-Cameroun et conséquences pour l'agriculture : Actes $\mathrm{du}$ colloque, Savanes africaines : des espaces en mutation, des acteurs face à de nouveaux défis, Garoua, Cameroun, Jamin JY, Seiny Boukar L (Eds), Prasac, N'Djamena, Tchad. Cirad : Montpellier, France ; 9 p.

MINEF. 2000. Inventaire ornithologique de la réserve forestière du Mayo-Louti et du parc national de Mozogo-Gokoro, Rapport technique et synthèse de l'avifaune, club ornithologique du Cameroun Birdlife International, République du Cameroun, $16 \mathrm{p}$.

Ntoupka M. 1999. Impact des perturbations anthropiques (pâturage, feu, et coupe de bois) sur la dynamique de la savane arborée en zone soudano-sahélienne du Nord du Cameroun, thèse de Doctorat de l'université, Paul Valery-Montpellier III, $260 \mathrm{p}$.

SACART. 2008. Carte du Cameroun au 1/50000, Mokolo 4d.

Sandjong SRC. 2012. Etudes écologique et floristique du Parc National de MozogoGokoro (Cameroun): prospections préliminaires pour sa conservation et son aménagement. Thèse de Master, Université de Maroua, 83p.

Sonké B. 1998. Étude floristique et structurale des forêts de la réserve de faune du Dja (Cameroun). Thèse de Doctorat, Université Libre de Bruxelles, Faculté des Sciences, 267p.

Tchobsala, Amougou A, Mbolo M. 2010. Impact of wood cut on the structure and floristic diversity of vegetation in the peri-urban zone of Ngaoundéré (Cameroon). Journal of Ecology and the Natural Environment, 2(11): 235-258.

Wafo T. 2008. Les aires protégées de l'Extrême-Nord Cameroun, entre politiques de conservation et pratiques locales. Thèse de Doctorat, Université d'Orléans, Pôle Universités Centre Val de Loire, France, 325 p. 